CRYSTALLOGRAPHIC COMMUNICATIONS

ISSN 2056-9890

Received 18 November 2021

Accepted 17 January 2022

Edited by D. Chopra, Indian Institute of Science Education and Research Bhopal, India

Keywords: molecular structure; crystal structure; 2-(3-hydroxypropyl)benzimidazole; hydrogen bond; Hirshfeld analysis; periodic calculations.

CCDC references: 2142658; 2142657

Supporting information: this article has supporting information at journals.iucr.org/e
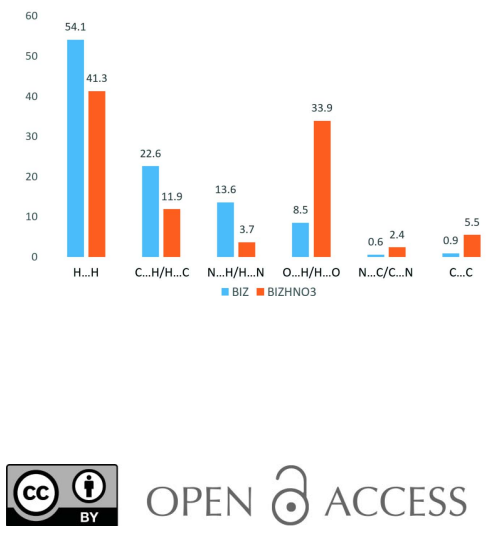

\section{The molecular and crystal structures of 2-(3-hydroxypropyl)benzimidazole and its nitrate salt}

\author{
Dilnoza Rakhmonova, ${ }^{\mathrm{a} *}$ Zukhra Kadirova, ${ }^{\mathrm{a}}$ Batirbay Torambetov, ${ }^{\mathrm{a}}$ Shakhnoza \\ Kadirova, ${ }^{a}$ Jamshid Ashurov ${ }^{b}$ and Svitlana Shishkina ${ }^{c}$
}

aNational University of Uzbekistan named after Mirzo Ulugbek, 4 University St, Tashkent, 100174, Uzbekistan, ' Institute
of Bioorganic Chemistry, Academy of Sciences of Uzbekistan, M. Ulugbek Str, 83, Tashkent, 700125, Uzbekistan, and
${ }^{\mathbf{c} S t a t e ~ S c i e n t i f i c ~ I n s t i t u t i o n ~ " I n s t i t u t e ~ f o r ~ S i n g l e ~ C r y s t a l s " ~ o f ~ N a t i o n a l ~ A c a d e m y ~ o f ~ S c i e n c e s ~ o f ~ U k r a i n e, ~} 60$ Nauky ave.,
61001 Kharkiv, Ukraine. *Correspondence e-mail: d.rakhmonova81@mail.ru

2-(3-Hydroxypropyl)- $1 H$-benzimidazole, $\mathrm{C}_{10} \mathrm{H}_{12} \mathrm{~N}_{2} \mathrm{O}$, which has potential biological activity, can be used as a ligand for complexation with metals. This compound is an electron donor, due to the lone pair of the nitrogen atom in the imidazole ring. This nitrogen atom also acts as a proton acceptor. In the crystalline phase, the nitrate salt, namely, 2-(3-hydroxypropyl)- $1 H$-benzimidazol-3-ium nitrate, $\mathrm{C}_{10} \mathrm{H}_{13} \mathrm{~N}_{2} \mathrm{O}^{+} \cdot \mathrm{NO}_{3}{ }^{-}$, has been studied. The protonation of the 2-(3-hydroxypropyl)benzimidazole unit results in significant delocalization of the electron density within the imidazole ring. The salt formation leads to variations in the intermolecular interactions, which were studied by analysis of the Hirshfeld surfaces and two-dimensional fingerprint plots.

\section{Chemical context}

Benzimidazole derivatives and their complex compounds possess a wide spectrum of biological activity (Salahuddin et al., 2012), including antibacterial (Chkirate et al., 2020), antifungal (Khabnadideh et al., 2012), antiviral (Kharitonova et al., 2017), antiparasitic (Katti et al., 2019), anti-inflammatory and analgesic (Gaba et al., 2014) activities.

Nitrogen-containing heterocycles can be lone-pair donors, forming complex compounds with a metal; in some, the nitrogen heterocycle binds to the metal atom (Mottillo et al., 2015). The lone pair of the cyclic nitrogen atom can be protonated, forming an organic cation (Yan et al., 2009; Yu et al., 2007, Bayar et al., 2018; Chen et al., 2010). It has been shown (Pilipenko \& Tananaiko, 1983) that compounds containing a protonated cation are formed as a result of the combination with counter-ions. Such compounds, also called ionic associates, are intermediate compounds between simple salts and complex (coordination) compounds. They have properties similar to those of mixed-ligand complexes, although the properties of the compound as a whole depends on many factors.

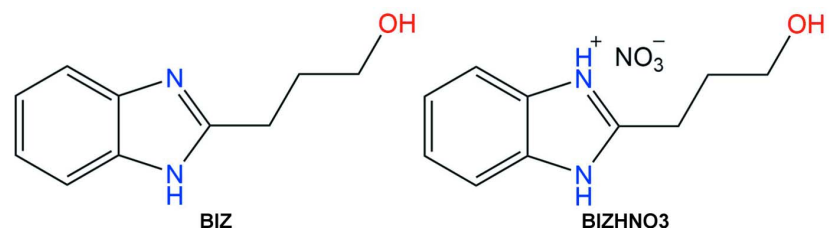

In the present paper we report the molecular and crystal structures of 2-(3-hydroxypropyl)benzimidazole (BIZ) and its 


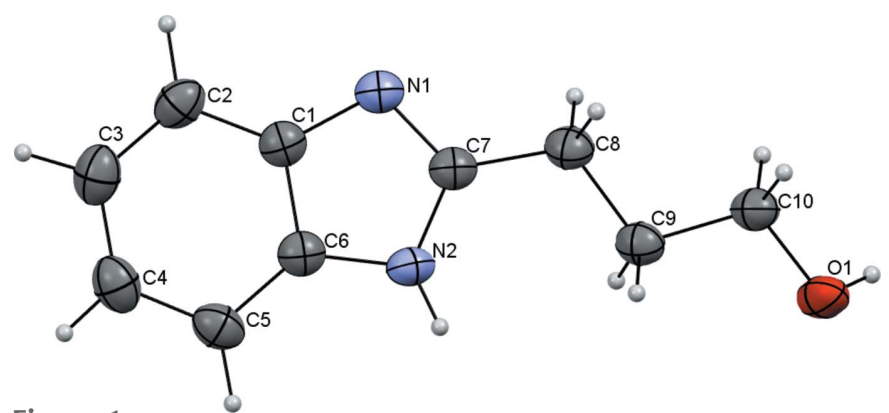

Figure 1

Molecular structure of the neutral 2-(3-hydroxypropyl)benzimidazole molecule in the BIZ structure. Displacement ellipsoids are shown at the $50 \%$ probability level.

nitrate salt (BIZHNO3), which were determined to study the influence of protonation.

\section{Structural commentary}

Analysis of the molecular structures of the title compounds revealed that the $\mathrm{C} 7-\mathrm{N} 1$ and $\mathrm{C} 7-\mathrm{N} 2$ bonds have different lengths $[\mathrm{N} 1-\mathrm{C} 7=1.322$ (4) $\AA$ and N2-C7 = 1.352 (4) $\mathrm{A}]$ in the neutral BIZ molecule (Fig. 1) but are equal within standard uncertainties $[\mathrm{N} 1-\mathrm{C} 7=1.329(2) \AA$ and $\mathrm{N} 2-\mathrm{C} 7=$ 1.331 (2) $\AA$ ] in its protonated form in BIZHNO3 (Fig. 2). Such a delocalization of the electron density during protonation allows the structure of protonated BIZ molecule to be described as a superposition of two resonance structures, as shown in the scheme below.

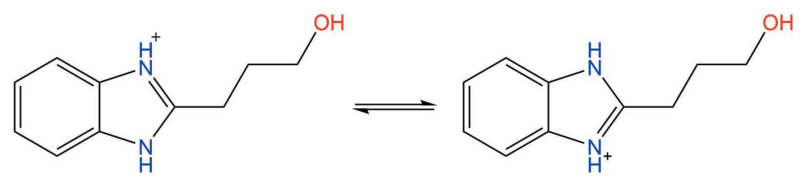

The neutral and protonated BIZ molecules differ in the conformation of the hydroxyalkyl substituent (Figs. 1 and 2).
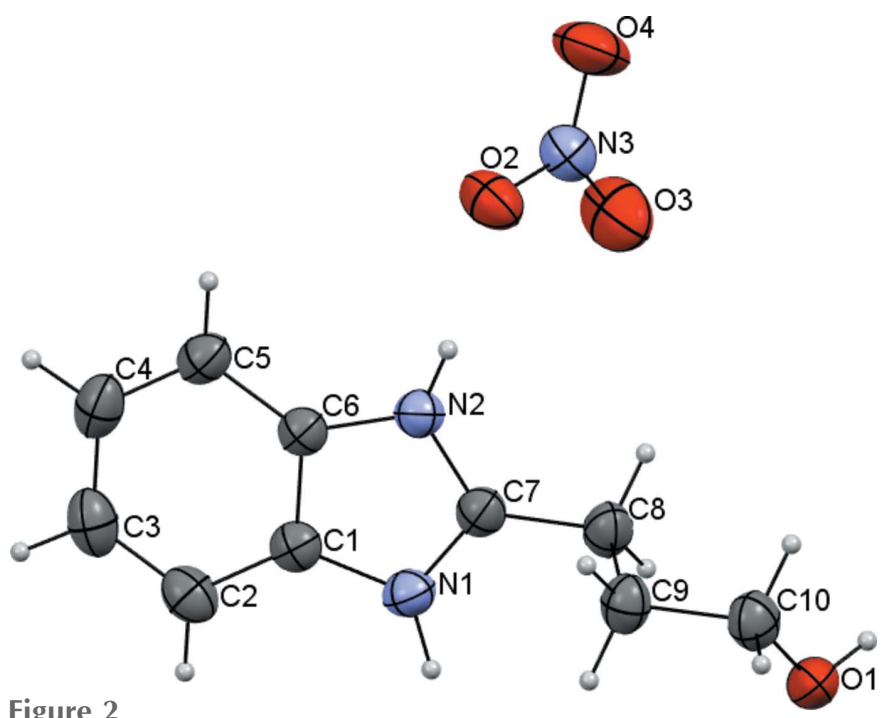

Molecular structure of the 2-(3-hydroxypropyl)benzimidazole nitrate salt in the BIZHNO3 structure. Displacement ellipsoids are shown at the $50 \%$ probability level.
Table 1

Hydrogen-bond geometry $\left(\AA,^{\circ}\right)$ for BIZ.

\begin{tabular}{lllll}
\hline$D-\mathrm{H} \cdots A$ & $D-\mathrm{H}$ & $\mathrm{H} \cdots A$ & $D \cdots A$ & $D-\mathrm{H} \cdots A$ \\
\hline $\mathrm{N} 2-\mathrm{H} 2 N \cdots \mathrm{O} 1^{\mathrm{i}}$ & $0.84(3)$ & $1.95(3)$ & $2.772(3)$ & $165(3)$ \\
$\mathrm{O} 1-\mathrm{H} 1 \cdots \mathrm{N} 1^{\mathrm{ii}}$ & $0.85(4)$ & $1.90(4)$ & $2.741(3)$ & $169(4)$ \\
$\mathrm{C} 3-\mathrm{H} 3 \cdots \mathrm{C} 3^{\text {iii }}$ & $0.97(4)$ & $2.87(4)$ & $3.770(4)$ & $154(3)$ \\
\hline Symmetry codes: & (i) & $x+\frac{1}{2},-y+\frac{1}{2},-z+1 ;$ & (ii) & $-x+\frac{1}{2},-y+1, z+\frac{1}{2} ; \quad$ (iii) \\
$x+\frac{1}{2},-y+\frac{1}{2},-z$. & & &
\end{tabular}

Table 2

Hydrogen-bond geometry $\left(\AA,^{\circ}\right)$ for BIZHNO3.

\begin{tabular}{lllll}
\hline$D-\mathrm{H} \cdots A$ & $D-\mathrm{H}$ & $\mathrm{H} \cdots A$ & $D \cdots A$ & $D-\mathrm{H} \cdots A$ \\
\hline $\mathrm{N} 2-\mathrm{H} 2 N \cdots \mathrm{O} 2$ & $0.86(2)$ & $1.90(2)$ & $2.755(2)$ & $173(2)$ \\
$\mathrm{N} 1-\mathrm{H} 1 N \cdots \mathrm{O} 1^{\mathrm{i}}$ & $0.91(2)$ & $1.78(2)$ & $2.696(2)$ & $177(2)$ \\
$\mathrm{O} 1-\mathrm{H} 1 O \cdots 2^{\mathrm{ii}}$ & $0.90(3)$ & $2.06(3)$ & $2.866(2)$ & $149(3)$ \\
$\mathrm{O} 1-\mathrm{H} 1 O \cdots \mathrm{O} 4^{\mathrm{ii}}$ & $0.90(3)$ & $2.14(3)$ & $2.951(3)$ & $150(3)$ \\
$\mathrm{C} 5-\mathrm{H} 5 \cdots \mathrm{O} 4^{\mathrm{iii}}$ & 0.93 & 2.43 & $3.251(3)$ & 147 \\
\hline
\end{tabular}

Symmetry codes: (i) $-x+2,-y+1,-z+1$; (ii) $\quad-x+\frac{3}{2}, y+\frac{1}{2},-z+\frac{3}{2}$; (iii) $-x+\frac{1}{2}, y-\frac{1}{2},-z+\frac{3}{2}$.

In the neutral BIZ molecule, the hydroxyalkyl substituent is almost coplanar to the benzimidazole fragment [the N2-C7C8-C9 torsion angle is $15.3(4)^{\circ}$. The hydroxyalkyl substituent has an all-trans conformation [C7-C8-C9-C10 and $\mathrm{C} 8-\mathrm{C} 9-\mathrm{C} 10-\mathrm{O} 1=-179.8(3)$ and $-178.7(3)^{\circ}$, respectively]. In the protonated BIZ molecule, the hydroxyalkyl substituent is rotated orthogonally to the benzimidazole fragments $\left[\mathrm{N} 2-\mathrm{C} 7-\mathrm{C} 8-\mathrm{C} 9=103.1(2)^{\circ}\right]$ and has an $a p-,-s c$ conformation $[\mathrm{C} 7-\mathrm{C} 8-\mathrm{C} 9-\mathrm{C} 10$ and $\mathrm{C} 8-\mathrm{C} 9-$ $\mathrm{C} 10-\mathrm{O} 1=-179.3(2)$ and $-62.3(2)^{\circ}$, respectively].

\section{Supramolecular features}

In the crystal, BIZ molecules are linked by $\mathrm{O}-\mathrm{H} \cdots \mathrm{N}$ and $\mathrm{N}-\mathrm{H} \cdots \mathrm{O}$ hydrogen bonds (Table 1). The zigzag chains formed by the $\mathrm{N}-\mathrm{H} \cdots \mathrm{O}$ hydrogen bonds propagate in the [100] direction (Fig. 3, on the left). These chains are connected by $\mathrm{O}-\mathrm{H} \cdots \mathrm{N}$ hydrogen bonds in the [010] and [001] directions (Fig. 3, on the right; the chains are highlighted in blue). In addition, weak $\mathrm{C} 3-\mathrm{H} \cdots \mathrm{C} 3(\pi)$ interactions (Table 1 ) are observed between the BIZ molecules.

In the crystal of the nitrate salt, the protonated BIZ molecules are connected by $\mathrm{N}-\mathrm{H} \cdots \mathrm{O}$ hydrogen bonds (Table 2),
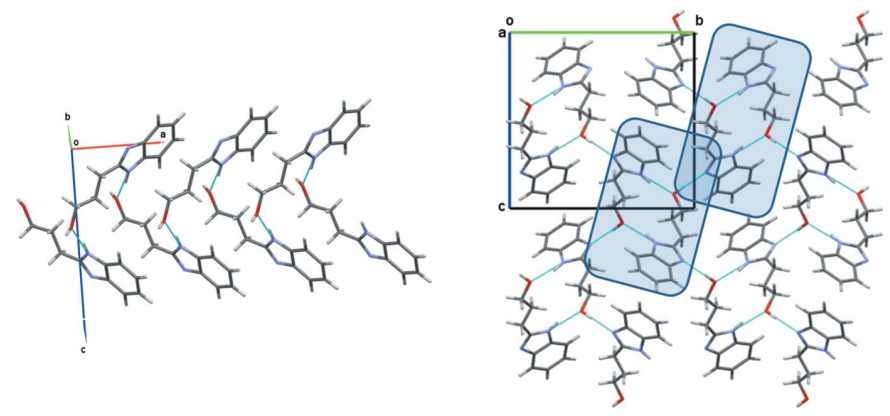

Figure 3

Crystal packing of the neutral molecules in the BIZ structure. Projection in the [100] direction. 


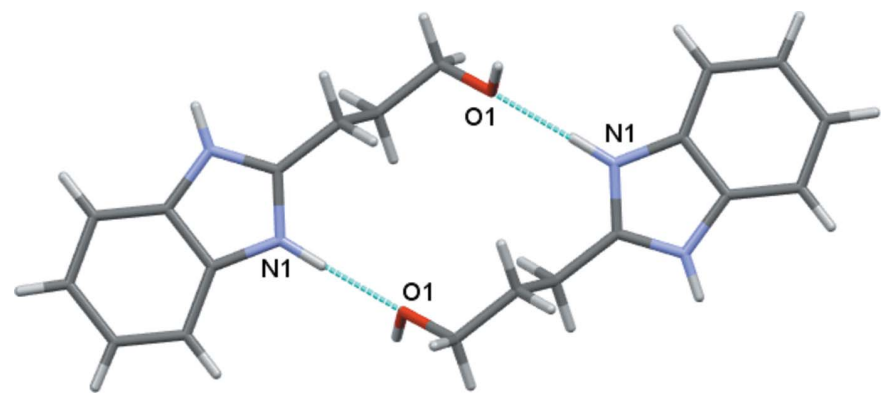

Figure 4

Hydrogen-bonded centrosymmetric dimer of the cations in the nitrate salt. Hydrogen bonds are shown by the cyan lines.

forming centrosymmetric dimers (Fig. 4). These dimers are linked by the bridging nitrate anions in the [001] direction via $\mathrm{N}-\mathrm{H} \cdots \mathrm{O}, \mathrm{O}-\mathrm{H} \cdots \mathrm{O}$ and $\mathrm{C}-\mathrm{H} \cdots \mathrm{O}$ hydrogen bonds (Fig. 5). Stacking interactions of the head-to-tail type between the imidazole rings of $\mathrm{BIZH}^{+}$molecules are observed in the [010] direction, the distance between $\pi$-systems being 3.502 (2) $\AA$.

\section{Hirshfeld surface analysis}

Hirshfeld surface analysis (Turner et al., 2017) is one of the modern methods allowing intermolecular interactions to be studied in a more analytical way. This method appears to be effective for comparing the capability of the neutral BIZ molecule and its protonated form to participate in intermolecular interactions of different types. The Hirshfeld surfaces were calculated for the $\mathrm{BIZ}$ and $\mathrm{BIZH}^{+}$molecules using a standard high surface resolution, mapped over $d_{\text {norm }}$ (Fig. 6). Bright-red spots are observed for all the donors and acceptors of strong hydrogen bonds in the two structures under study, indicating their participation in intermolecular interactions. It should be noted that the bright-red spot on the $\mathrm{N} 1$ atom in the BIZ molecule indicates its capability to be protonated or participate in complexation with a metal.

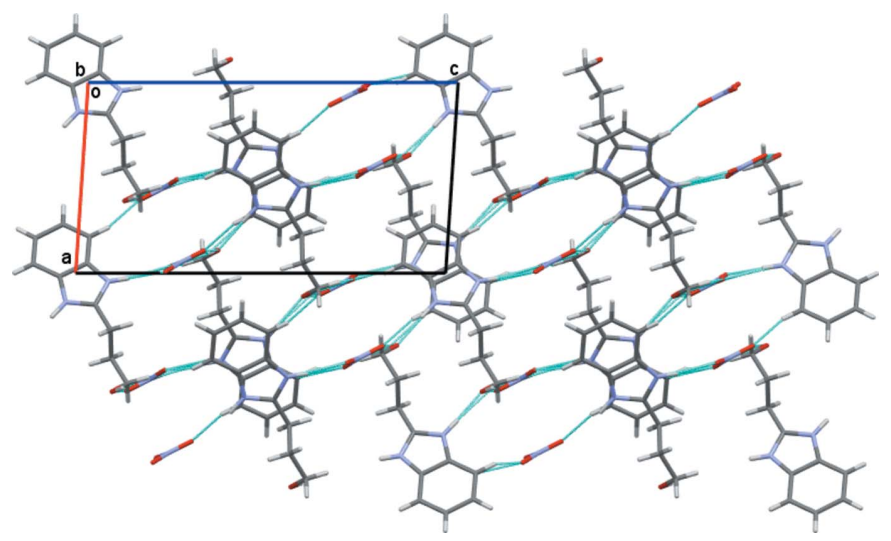

Figure 5

Crystal packing of the 2-(3-hydroxypropyl)benzimidazole nitrate salt in the BIZHNO3 structure. Projection in the [010] direction. Hydrogen bonds are shown by cyan lines.
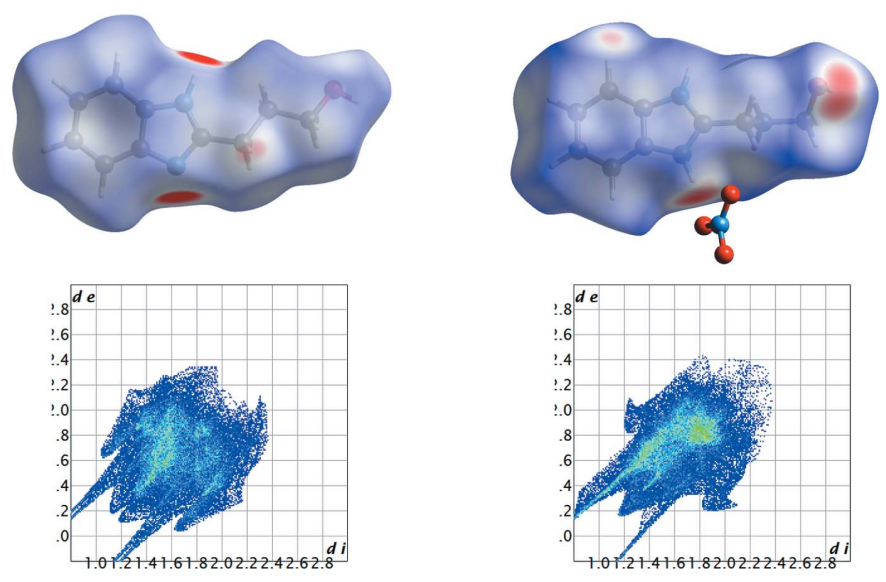

Figure 6

Hirshfeld surfaces mapped over $d_{\text {norm }}$ (top) and two-dimensional fingerprint plots (bottom) of the neutral 2-(3-hydroxypropyl)benzimidazole molecule and its protonated cation in the structures of BIZ and BIZHNO3.

The two-dimensional fingerprint plots constructed for the $\mathrm{BIZ}$ and $\mathrm{BIZH}^{+}$molecules show that the hydrogen bonds are stronger in the structure of the nitrate salt (see the sharp spikes in Fig. 6). To compare intermolecular interactions of different types in the structures under study, we have analysed their contributions to the total Hirshfeld surfaces (Fig. 7). As can be seen from the histogram, the protonation of the BIZ molecule and presence of the nitrate anion results in a significant increase of the contribution of $\mathrm{O} \cdots \mathrm{H} / \mathrm{H} \cdots \mathrm{O}$ interactions associated with $X-\mathrm{H} \cdots \mathrm{O}$ hydrogen bonds. In addition, the contributions of $\mathrm{N} \cdots \mathrm{C} / \mathrm{C} \cdots \mathrm{N}$ and $\mathrm{C} \cdots \mathrm{C}$ interactions indicate that stacking between imidazole rings also increases in the BIZHNO3 structure (Fig. 7). A significant decrease in the contribution of $\mathrm{N} \cdots \mathrm{H} / \mathrm{H} \cdots \mathrm{N}$ interactions $(X-\mathrm{H} \cdots \mathrm{N}$ bonding) in the BIZHNO3 structure can be explained by the protonation of the $\mathrm{N} 1$ atom, which participates as proton acceptor of hydrogen bonds in the BIZ structure. The different contributions of $\mathrm{C} \cdot \mathrm{H} / \mathrm{H} \cdots \mathrm{C}$ interactions associated with $X-\mathrm{H} \cdots \mathrm{C}(\pi)$ hydrogen bonds coin-

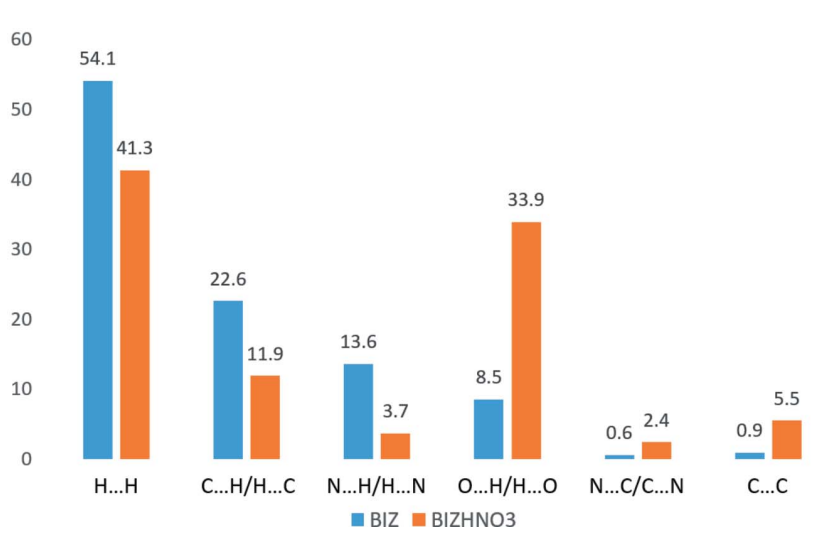

Figure 7

Relative contributions of the strongest intermolecular interactions (in \%) to the total Hirshfeld surface of the neutral molecule and its cation in the structures of BIZ and BIZHNO3. 
Table 3

Experimental details.

\begin{tabular}{|c|c|c|}
\hline & $\mathrm{BIZ}$ & BIZHNO3 \\
\hline Chemical formula & $\mathrm{C}_{10} \mathrm{H}_{12} \mathrm{~N}_{2} \mathrm{O}$ & $\mathrm{C}_{10} \mathrm{H}_{13} \mathrm{~N}_{2} \mathrm{O}^{+} \cdot \mathrm{NO}_{3}^{-}$ \\
\hline$M_{\mathrm{r}}$ & 176.22 & 239.23 \\
\hline Temperature $(\mathrm{K})$ & 293 & 293 \\
\hline$a, b, c(\AA)$ & $5.852(2), 12.437(3), 12.444(3)$ & $8.5100(3), 8.2525(4), 16.5130(7)$ \\
\hline$\alpha, \beta, \gamma\left({ }^{\circ}\right)$ & $90,90,90$ & $90,93.760(4), 90$ \\
\hline$\mu\left(\mathrm{mm}^{-1}\right)$ & 0.69 & 0.91 \\
\hline Crystal size $(\mathrm{mm})$ & $0.12 \times 0.10 \times 0.08$ & $0.12 \times 0.10 \times 0.08$ \\
\hline \multicolumn{3}{|l|}{ Data collection } \\
\hline Diffractometer & Oxford Diffraction Xcallibur, Ruby & Oxford Diffraction Xcalibur, Ruby \\
\hline Absorption correction & $\begin{array}{l}\text { Multi-scan (CrysAlis PRO; Oxford Diffraction, } \\
\text { 2009) }\end{array}$ & $\begin{array}{l}\text { Multi-scan (CrysAlis PRO; Oxford Diffraction, } \\
\text { 2009) }\end{array}$ \\
\hline$\theta_{\max }\left({ }^{\circ}\right)$ & 62.0 & 75.8 \\
\hline$(\sin \theta / \lambda)_{\max }\left(\AA^{-1}\right)$ & 0.573 & 0.629 \\
\hline \multicolumn{3}{|l|}{ Refinement } \\
\hline$R\left[F^{2}>2 \sigma\left(F^{2}\right)\right], w R\left(F^{2}\right), S$ & $0.039,0.091,1.06$ & $0.044,0.128,1.04$ \\
\hline No. of reflections & 1371 & 2345 \\
\hline No. of parameters & 166 & 167 \\
\hline $\mathrm{H}$-atom treatment & All H-atom parameters refined & $\begin{array}{l}\mathrm{H} \text { atoms treated by a mixture of independent } \\
\text { and constrained refinement }\end{array}$ \\
\hline$\Delta \rho_{\max }, \Delta \rho_{\min }\left(\mathrm{e} \AA^{-3}\right)$ & $0.11,-0.19$ & $0.16,-0.15$ \\
\hline Absolute structure & $\begin{array}{l}\text { Flack } x \text { determined using } 428 \text { quotients } \\
\qquad\left[\left(I^{+}\right)-\left(I^{-}\right)\right] /\left[\left(I^{+}\right)+\left(I^{-}\right)\right] \text {(Parsons } \text { et al., 2013) }\end{array}$ & - \\
\hline Absolute structure parameter & $0.1(3)$ & - \\
\hline
\end{tabular}

Computer programs: CrysAlis PRO (Oxford Diffraction, 2009), SHELXS97 (Sheldrick, 2008), SHELXL2016/6 (Sheldrick, 2015) and OLEX2 (Dolomanov et al., 2009).

cide with the presence of a $\mathrm{C}-\mathrm{H} \cdots \mathrm{C}(\pi)$ hydrogen bond in the BIZ structure (Table 1) and the absence of similar interactions in the BIZHNO3 structure (Table 2). The nitrate anions act as bridging moieties in the BIZHNO3 structure, which results in an increase in the distances between $\mathrm{BIZH}^{+}$molecules. This fact can explain the decrease in the contribution of $\mathrm{H} \cdots \mathrm{H}$ interactions in the BIZHNO3 structure (Fig. 7).

\section{Database survey}

A search of the Cambridge Structural Database (CSD, version 5.42, update of November 2020; Groom et al., 2016) revealed three structures containing the BIZ molecule [refcodes FIYXAN and FIYXER (Elmali et al., 2005) and RIYNUL (Zhao et al., 2019)]. Two of these structures (FIYXAN and FIYXER) contain protonated BIZ molecules, which form salts with $\mathrm{PtCl}_{4}{ }^{2-}$ or $\mathrm{PtCl}_{6}{ }^{2-}$ anions. In the RIYNUL structure, the BIZ molecule forms a coordination bond with the $\mathrm{Cd}$ atom.

In addition, three structures with a close analogue of the BIZ molecule containing a carboxylic group instead of a hydroxyl group were found in the CSD [refcodes JOQROZ (Fu et al., 2016), NOVCEI (Liu et al., 2015) and TILGOL (Zeng et al., 2007)]. In all of these structures, the organic ligand forms an $\mathrm{N}-M^{+}$coordination bond with participation of the $\mathrm{N} 2$ atom of the imidazole ring.

\section{Synthesis and crystallization}

All chemicals were obtained from commercial sources and used directly without further purification. 1,2-Phenylenediamine (2.16 g, $0.02 \mathrm{~mol}$ ) was dissolved in hydrochloric acid $(25 \mathrm{~mL}, 4 \mathrm{M})$ at $373 \mathrm{~K}$, and $\gamma$-hydroxybutyric acid $(2.82 \mathrm{~g}$, $0.02 \mathrm{~mol}$ ) was added to the solution. The mixture was heated with reflux for $6 \mathrm{~h}$ at $398 \mathrm{~K}$. After cooling to room temperature, the mixture was neutralized using $\mathrm{NaOH}(\mathrm{pH} 7-9)$. The product was dissolved in aqueous ethanol and treated with activated carbon for purification. The 2-hydroxypropylbenzimidazole precipitate was filtered off and dried in air. Pale-beige single crystals of the title compound suitable for $\mathrm{X}$-ray diffraction analysis were recrystallized from ethanol solution by slow evaporation, yield $80 \%$, m.p. 437 K.

Synthesis of the $\left[\mathrm{BIZH}^{+}\right] \mathrm{NO}_{3}{ }^{-}$salt:

A weighed portion of copper nitrate $\left(3 \times 10^{-3} \mathrm{~mol}\right)$ was dissolved in a minimum amount of water and mixed with an alcoholic saturated solution of the ligand $\left(6 \times 10^{-3} \mathrm{~mol}\right)$ while heating in a water bath. The solution turned green. The solution was then acidified with nitric acid to $\mathrm{pH} 5$ to prevent 
the precipitation of hydroxides. The reaction was carried out for 40 minutes while heating in a water bath, after which the reaction mixture was allowed to crystallize. After three days, the precipitated light-yellow crystals were separated, washed with ethanol, and dried in air. The product yield was $62 \%$, m.p. 371-373 K.

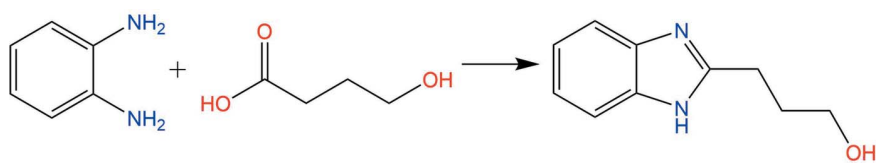

\section{Refinement}

Crystal data, data collection and structure refinement details are summarized in Table 3. All hydrogen atoms were located in difference-Fourier maps. All of the hydrogen atoms in the $\mathrm{BIZ}$ structure and $\mathrm{H}$ atoms participating in strong hydrogen bonds in the BIZHNO3 structure were refined using an isotropic approximation. Other hydrogen atoms in the BIZHNO3 structure were refined as riding with $\mathrm{Csp} p^{2}-\mathrm{H}=$ $0.97 \AA, U_{\text {iso }}(\mathrm{H})=1.2 U_{\text {eq }}(\mathrm{C})$ for the methylene fragments or $\mathrm{Car}-\mathrm{H}=0.93 \AA, U_{\text {iso }}(\mathrm{H})=1.2 U_{\text {eq }}(\mathrm{C})$ for the aromatic rings.

\section{References}

Bayar, I., Khedhiri, L., Soudani, S., Lefebvre, F., Ferretti, V. \& Ben Nasr, C. (2018). J. Mol. Struct. 1161, 486-496.

Chen, S. H., Yang, F. R., Wang, M. T. \& Wang, N. (2010). C. R. Chim. 13, 1391-1396.

Chkirate, K., Karrouchi, K., Dege, N., Kheira Sebbar, N., Ejjoummany, A., Radi, S., Adarsh, N. N., Talbaoui, A., Ferbinteanu, M., Essassi, E. M. \& Garcia, Y. (2020). New J. Chem. 44, 2210-2221.

Dolomanov, O. V., Bourhis, L. J., Gildea, R. J., Howard, J. A. K. \& Puschmann, H. (2009). J. Appl. Cryst. 42, 339-341.

Elmali, A., Elerman, Y., Eren, G., Gümüş, F. \& Svoboda, I. (2005). Z. Naturforsch. Teil B, 60, 164-168.
Fu, W.-W., Liu, Y.-Y., Zhang, Y.-H., Xu, L., Zhang, J.-L. \& Wei, H.-B. (2016). Synth. React. Inorg. Met.-Org. Nano-Met. Chem. 46, 18571860.

Gaba, M., Singh, S. \& Mohan, C. (2014). Eur. J. Med. Chem. 76, 494505.

Groom, C. R., Bruno, I. J., Lightfoot, M. P. \& Ward, S. C. (2016). Acta Cryst. B72, 171-179.

Katti, S. A., Desai, K. S. \& Loharkar, S. V. (2019). WJPR. 8, 11411151.

Khabnadideh, S., Rezaei, Z., Pakshir, K., Zomorodian, K. \& Ghafari, N. (2012). Res. Pharm. Sci. 7, 65-72.

Kharitonova, M. I., Antonov, K. V., Fateev, I. V., Berzina, M. Ya., Kaushin, A. L., Paramonov, A. S., Kotovskaya, S. K., Andronova, V. L., Konstantinova, I. D., Galegov, G. A., Charushin, V. N. \& Miroshnikov, A. I. (2017). Synthesis, 49, 1043-1052.

Liu, Z., Zheng, S. \& Feng, S. (2015). Acta Cryst. E71, m5-m6.

Mottillo, C. \& Friščić, T. (2015). Chem. Commun. 51, 8924-8927.

Oxford Diffraction (2009). CrysAlis PRO. Oxford Diffraction Ltd, Yarnton, England.

Parsons, S., Flack, H. D. \& Wagner, T. (2013). Acta Cryst. B69, 249259.

Pilipenko, A. T. \& Tananaiko, M. M. (1983). Mixed-ligand and mixedmetal complexes and their application in analytical chemistry. Moscow: Chemistry.

Salahuddin, Shaharyar, M. \& Mazumder, A. (2012). Arab. J. Chem. 10, S157-S173.

Sheldrick, G. M. (2008). Acta Cryst. A64, 112-122.

Sheldrick, G. M. (2015). Acta Cryst. C71, 3-8.

Turner, M. J., McKinnon, J. J., Wolff, S. K., Grimwood, D. J., Spackman, P. R., Jayatilaka, D. \& Spackman, M. A. (2017). Turner CrystalExplorer17. University of Western Australia. http://Hirshfeldsurface.net

Yan, Z. Z., Hou, N., Liang, H. D., Zhao, S. L. \& Tang, Y. (2009). X-ray Struct. Anal. Online, 25, 31-32.

Yu, Q.-Y., Cai, Y.-P. \& Ng, S. W. (2007). Acta Cryst. E63, o881-o882. Zeng, M.-H., Yao, M.-X., Liang, H. \& Ng, S. W. (2007). J. Coord. Chem. 60, 1983-1987.

Zhao, Y., Han, X., Yu, F., Wei, D., Cheng, Q., Meng, X., Ding, J. \& Hou, H. (2019). Chem. Eur. J. 25, 5246-5250. 


\section{supporting information}

Acta Cryst. (2022). E78, 211-215 [https://doi.org/10.1107/S2056989022000585]

The molecular and crystal structures of 2-(3-hydroxypropyl)benzimidazole and its nitrate salt

Dilnoza Rakhmonova, Zukhra Kadirova, Batirbay Torambetov, Shakhnoza Kadirova, Jamshid Ashurov and Svitlana Shishkina

Computing details

For both structures, data collection: CrysAlis PRO (Oxford Diffraction, 2009); cell refinement: CrysAlis PRO (Oxford Diffraction, 2009); data reduction: CrysAlis PRO (Oxford Diffraction, 2009); program(s) used to solve structure:

SHELXS97 (Sheldrick, 2008); program(s) used to refine structure: SHELXL2016/6 (Sheldrick, 2015); molecular graphics: OLEX2 (Dolomanov et al., 2009); software used to prepare material for publication: OLEX2 (Dolomanov et al., 2009).

2-(3-Hydroxypropyl)-1H-benzimidazole (BIZ)

Crystal data

$\mathrm{C}_{10} \mathrm{H}_{12} \mathrm{~N}_{2} \mathrm{O}$

$M_{r}=176.22$

Orthorhombic, $P 22_{1} 2_{1}$

$a=5.852(2) \AA$

$b=12.437(3) \AA$

$c=12.444(3) \AA$

$V=905.7(4) \AA^{3}$

$Z=4$

$F(000)=376$

Data collection

Oxford Diffraction Xcallibur, Ruby diffractometer

Radiation source: Enhance $(\mathrm{Cu}) \mathrm{X}$-ray Source Detector resolution: 10.2576 pixels $\mathrm{mm}^{-1}$ $\omega$ scans

Absorption correction: multi-scan (CrysAlisPro; Oxford Diffraction, 2009)

$T_{\min }=0.958, T_{\max }=1.000$

Refinement

Refinement on $F^{2}$

Least-squares matrix: full

$R\left[F^{2}>2 \sigma\left(F^{2}\right)\right]=0.039$

$w R\left(F^{2}\right)=0.091$

$S=1.06$

1371 reflections

166 parameters

0 restraints
$D_{\mathrm{x}}=1.292 \mathrm{Mg} \mathrm{m}^{-3}$

$\mathrm{Cu} K \alpha$ radiation, $\lambda=1.54178 \AA$

Cell parameters from 1839 reflections

$\theta=5.2-32.4^{\circ}$

$\mu=0.69 \mathrm{~mm}^{-1}$

$T=293 \mathrm{~K}$

Block, colorless

$0.12 \times 0.10 \times 0.08 \mathrm{~mm}$

3945 measured reflections

1371 independent reflections

1191 reflections with $I>2 \sigma(I)$

$R_{\text {int }}=0.029$

$\theta_{\max }=62.0^{\circ}, \theta_{\min }=5.0^{\circ}$

$h=-6 \rightarrow 6$

$k=-13 \rightarrow 11$

$l=-12 \rightarrow 14$

Primary atom site location: difference Fourier map

Secondary atom site location: difference Fourier map

Hydrogen site location: difference Fourier map

All H-atom parameters refined

$w=1 /\left[\sigma^{2}\left(F_{\mathrm{o}}^{2}\right)+(0.0573 P)^{2}\right]$

where $P=\left(F_{\mathrm{o}}^{2}+2 F_{\mathrm{c}}{ }^{2}\right) / 3$

$(\Delta / \sigma)_{\max }<0.001$ 


$$
\Delta \rho_{\max }=0.11 \text { e } \AA^{-3}
$$

$\Delta \rho_{\min }=-0.19$ e $\AA^{-3}$
Absolute structure: Flack $x$ determined using 428 quotients $\left[\left(I^{+}\right)-(I)\right] /\left[\left(I^{+}\right)+(I)\right]$ (Parsons et al., 2013)

Absolute structure parameter: $0.1(3)$

\section{Special details}

Geometry. All esds (except the esd in the dihedral angle between two 1.s. planes) are estimated using the full covariance matrix. The cell esds are taken into account individually in the estimation of esds in distances, angles and torsion angles; correlations between esds in cell parameters are only used when they are defined by crystal symmetry. An approximate (isotropic) treatment of cell esds is used for estimating esds involving l.s. planes.

Fractional atomic coordinates and isotropic or equivalent isotropic displacement parameters $\left(\AA^{2}\right)$

\begin{tabular}{lllll}
\hline & $x$ & $y$ & $z$ & $U_{\text {iso }} / U_{\text {eq }}$ \\
\hline O1 & $-0.0261(5)$ & $0.38855(16)$ & $0.5824(2)$ & $0.0635(7)$ \\
H1 & $-0.080(6)$ & $0.439(3)$ & $0.621(3)$ & $0.083(14)^{*}$ \\
N1 & $0.6471(4)$ & $0.43719(19)$ & $0.2047(2)$ & $0.0470(6)$ \\
N2 & $0.6271(4)$ & $0.2901(2)$ & $0.3056(2)$ & $0.0457(7)$ \\
H2N & $0.587(5)$ & $0.243(2)$ & $0.350(3)$ & $0.045(9)^{*}$ \\
C1 & $0.8204(5)$ & $0.3668(2)$ & $0.1742(2)$ & $0.0454(7)$ \\
C2 & $0.9878(6)$ & $0.3766(3)$ & $0.0962(3)$ & $0.0559(9)$ \\
H2 & $0.994(6)$ & $0.444(3)$ & $0.051(3)$ & $0.068(10)^{*}$ \\
C3 & $1.1430(6)$ & $0.2942(3)$ & $0.0847(3)$ & $0.0631(9)$ \\
H3 & $1.268(7)$ & $0.297(3)$ & $0.034(3)$ & $0.076(11)^{*}$ \\
C4 & $1.1335(6)$ & $0.2037(3)$ & $0.1499(3)$ & $0.0616(10)$ \\
H4 & $1.232(6)$ & $0.148(3)$ & $0.144(3)$ & $0.081(12)^{*}$ \\
C5 & $0.9683(6)$ & $0.1915(3)$ & $0.2277(3)$ & $0.0555(9)$ \\
H5 & $0.961(6)$ & $0.128(3)$ & $0.274(3)$ & $0.075(11)^{*}$ \\
C6 & $0.8096(5)$ & $0.2745(2)$ & $0.2377(2)$ & $0.0446(7)$ \\
C7 & $0.5365(5)$ & $0.3875(2)$ & $0.2830(2)$ & $0.0427(7)$ \\
C8 & $0.3317(6)$ & $0.4331(3)$ & $0.3370(3)$ & $0.0505(8)$ \\
H8A & $0.369(6)$ & $0.511(3)$ & $0.353(3)$ & $0.076(11)^{*}$ \\
H8B & $0.206(6)$ & $0.431(3)$ & $0.286(3)$ & $0.059(9)^{*}$ \\
C9 & $0.2555(6)$ & $0.3800(3)$ & $0.4403(3)$ & $0.0485(8)$ \\
H9A & $0.376(6)$ & $0.382(3)$ & $0.493(3)$ & $0.055(9)^{*}$ \\
H9B & $0.220(5)$ & $0.307(3)$ & $0.428(3)$ & $0.049(8)^{*}$ \\
C10 & $0.0467(6)$ & $0.4349(3)$ & $0.4844(3)$ & $0.0507(8)$ \\
H10A & $0.073(5)$ & $0.512(3)$ & $0.495(3)$ & $0.051(8)^{*}$ \\
H10B & $-0.086(6)$ & $0.432(3)$ & $0.432(3)$ & $0.069(10)^{*}$ \\
& & & &
\end{tabular}

Atomic displacement parameters $\left(\AA^{2}\right)$

\begin{tabular}{lllllll}
\hline & $U^{11}$ & $U^{22}$ & $U^{33}$ & $U^{12}$ & $U^{13}$ & $U^{23}$ \\
\hline O1 & $0.0956(18)$ & $0.0401(12)$ & $0.0547(14)$ & $0.0140(12)$ & $0.0202(13)$ & $0.0041(11)$ \\
N1 & $0.0527(15)$ & $0.0425(12)$ & $0.0457(15)$ & $-0.0018(11)$ & $-0.0032(13)$ & $0.0034(11)$ \\
N2 & $0.0518(15)$ & $0.0398(13)$ & $0.0454(16)$ & $-0.0046(11)$ & $-0.0032(12)$ & $0.0091(12)$ \\
C1 & $0.0476(17)$ & $0.0427(15)$ & $0.0459(18)$ & $-0.0053(14)$ & $-0.0035(14)$ & $-0.0040(13)$ \\
C2 & $0.064(2)$ & $0.0517(18)$ & $0.052(2)$ & $-0.0131(17)$ & $0.0045(16)$ & $-0.0032(16)$ \\
C3 & $0.060(2)$ & $0.066(2)$ & $0.064(2)$ & $-0.0104(18)$ & $0.0101(19)$ & $-0.016(2)$
\end{tabular}




\begin{tabular}{lllllll} 
C4 & $0.055(2)$ & $0.057(2)$ & $0.073(3)$ & $0.0039(17)$ & $-0.0013(18)$ & $-0.0160(19)$ \\
C5 & $0.059(2)$ & $0.0442(17)$ & $0.063(2)$ & $0.0022(15)$ & $-0.0082(18)$ & $-0.0027(17)$ \\
C6 & $0.0465(16)$ & $0.0425(15)$ & $0.0448(18)$ & $-0.0059(13)$ & $-0.0059(14)$ & $-0.0025(13)$ \\
C7 & $0.0468(16)$ & $0.0404(14)$ & $0.0409(16)$ & $-0.0043(13)$ & $-0.0073(14)$ & $-0.0007(13)$ \\
C8 & $0.0551(18)$ & $0.0509(17)$ & $0.0455(18)$ & $0.0022(16)$ & $-0.0049(16)$ & $0.0033(14)$ \\
C9 & $0.058(2)$ & $0.0418(18)$ & $0.046(2)$ & $0.0018(14)$ & $-0.0023(15)$ & $-0.0023(15)$ \\
C10 & $0.0614(19)$ & $0.0441(17)$ & $0.0467(19)$ & $0.0030(16)$ & $-0.0008(17)$ & $0.0035(15)$ \\
\hline
\end{tabular}

Geometric parameters $\left(\AA,{ }^{o}\right)$

\begin{tabular}{|c|c|c|c|}
\hline $\mathrm{O} 1-\mathrm{C} 10$ & $1.414(4)$ & $\mathrm{C} 4-\mathrm{C} 5$ & $1.376(5)$ \\
\hline $\mathrm{O} 1-\mathrm{H} 1$ & $0.85(4)$ & $\mathrm{C} 4-\mathrm{H} 4$ & $0.91(4)$ \\
\hline $\mathrm{N} 1-\mathrm{C} 7$ & $1.322(4)$ & $\mathrm{C} 5-\mathrm{C} 6$ & $1.394(4)$ \\
\hline $\mathrm{N} 1-\mathrm{C} 1$ & $1.393(4)$ & $\mathrm{C} 5-\mathrm{H} 5$ & $0.98(4)$ \\
\hline $\mathrm{N} 2-\mathrm{C} 7$ & $1.352(4)$ & $\mathrm{C} 7-\mathrm{C} 8$ & $1.486(5)$ \\
\hline $\mathrm{N} 2-\mathrm{C} 6$ & $1.376(4)$ & $\mathrm{C} 8-\mathrm{C} 9$ & $1.513(4)$ \\
\hline $\mathrm{N} 2-\mathrm{H} 2 \mathrm{~N}$ & $0.84(3)$ & $\mathrm{C} 8-\mathrm{H} 8 \mathrm{~A}$ & $1.02(4)$ \\
\hline $\mathrm{C} 1-\mathrm{C} 2$ & $1.385(4)$ & $\mathrm{C} 8-\mathrm{H} 8 \mathrm{~B}$ & $0.97(3)$ \\
\hline $\mathrm{C} 1-\mathrm{C} 6$ & $1.395(4)$ & $\mathrm{C} 9-\mathrm{C} 10$ & $1.503(5)$ \\
\hline $\mathrm{C} 2-\mathrm{C} 3$ & $1.377(5)$ & C9-H9A & $0.96(4)$ \\
\hline $\mathrm{C} 2-\mathrm{H} 2$ & $1.01(4)$ & C9-H9B & $0.94(3)$ \\
\hline $\mathrm{C} 3-\mathrm{C} 4$ & $1.389(5)$ & $\mathrm{C} 10-\mathrm{H} 10 \mathrm{~A}$ & $0.98(3)$ \\
\hline $\mathrm{C} 3-\mathrm{H} 3$ & $0.97(4)$ & $\mathrm{C} 10-\mathrm{H} 10 \mathrm{~B}$ & $1.01(4)$ \\
\hline $\mathrm{C} 10-\mathrm{O} 1-\mathrm{H} 1$ & $107(3)$ & $\mathrm{C} 5-\mathrm{C} 6-\mathrm{C} 1$ & $121.9(3)$ \\
\hline $\mathrm{C} 7-\mathrm{N} 1-\mathrm{C} 1$ & $105.3(2)$ & $\mathrm{N} 1-\mathrm{C} 7-\mathrm{N} 2$ & $112.3(3)$ \\
\hline $\mathrm{C} 7-\mathrm{N} 2-\mathrm{C} 6$ & $107.6(3)$ & $\mathrm{N} 1-\mathrm{C} 7-\mathrm{C} 8$ & $123.4(3)$ \\
\hline $\mathrm{C} 7-\mathrm{N} 2-\mathrm{H} 2 \mathrm{~N}$ & $131(2)$ & $\mathrm{N} 2-\mathrm{C} 7-\mathrm{C} 8$ & $124.3(3)$ \\
\hline $\mathrm{C} 6-\mathrm{N} 2-\mathrm{H} 2 \mathrm{~N}$ & $121(2)$ & $\mathrm{C} 7-\mathrm{C} 8-\mathrm{C} 9$ & $117.1(3)$ \\
\hline $\mathrm{C} 2-\mathrm{C} 1-\mathrm{N} 1$ & $130.6(3)$ & $\mathrm{C} 7-\mathrm{C} 8-\mathrm{H} 8 \mathrm{~A}$ & $106(2)$ \\
\hline $\mathrm{C} 2-\mathrm{C} 1-\mathrm{C} 6$ & $120.1(3)$ & $\mathrm{C} 9-\mathrm{C} 8-\mathrm{H} 8 \mathrm{~A}$ & $109(2)$ \\
\hline $\mathrm{N} 1-\mathrm{C} 1-\mathrm{C} 6$ & $109.3(3)$ & $\mathrm{C} 7-\mathrm{C} 8-\mathrm{H} 8 \mathrm{~B}$ & $107(2)$ \\
\hline $\mathrm{C} 3-\mathrm{C} 2-\mathrm{C} 1$ & $118.3(3)$ & $\mathrm{C} 9-\mathrm{C} 8-\mathrm{H} 8 \mathrm{~B}$ & $109(2)$ \\
\hline $\mathrm{C} 3-\mathrm{C} 2-\mathrm{H} 2$ & $123(2)$ & $\mathrm{H} 8 \mathrm{~A}-\mathrm{C} 8-\mathrm{H} 8 \mathrm{~B}$ & $109(3)$ \\
\hline $\mathrm{C} 1-\mathrm{C} 2-\mathrm{H} 2$ & $119(2)$ & $\mathrm{C} 10-\mathrm{C} 9-\mathrm{C} 8$ & $110.6(3)$ \\
\hline $\mathrm{C} 2-\mathrm{C} 3-\mathrm{C} 4$ & $121.1(4)$ & $\mathrm{C} 10-\mathrm{C} 9-\mathrm{H} 9 \mathrm{~A}$ & $109.7(19)$ \\
\hline $\mathrm{C} 2-\mathrm{C} 3-\mathrm{H} 3$ & $123(2)$ & $\mathrm{C} 8-\mathrm{C} 9-\mathrm{H} 9 \mathrm{~A}$ & $111(2)$ \\
\hline $\mathrm{C} 4-\mathrm{C} 3-\mathrm{H} 3$ & $116(2)$ & $\mathrm{C} 10-\mathrm{C} 9-\mathrm{H} 9 \mathrm{~B}$ & $108.7(19)$ \\
\hline $\mathrm{C} 5-\mathrm{C} 4-\mathrm{C} 3$ & $121.9(4)$ & $\mathrm{C} 8-\mathrm{C} 9-\mathrm{H} 9 \mathrm{~B}$ & $110.1(19)$ \\
\hline $\mathrm{C} 5-\mathrm{C} 4-\mathrm{H} 4$ & $115(2)$ & $\mathrm{H} 9 \mathrm{~A}-\mathrm{C} 9-\mathrm{H} 9 \mathrm{~B}$ & $107(3)$ \\
\hline $\mathrm{C} 3-\mathrm{C} 4-\mathrm{H} 4$ & $123(2)$ & $\mathrm{O} 1-\mathrm{C} 10-\mathrm{C} 9$ & $112.0(3)$ \\
\hline $\mathrm{C} 4-\mathrm{C} 5-\mathrm{C} 6$ & $116.7(3)$ & $\mathrm{O} 1-\mathrm{C} 10-\mathrm{H} 10 \mathrm{~A}$ & $109.1(19)$ \\
\hline $\mathrm{C} 4-\mathrm{C} 5-\mathrm{H} 5$ & $122(2)$ & $\mathrm{C} 9-\mathrm{C} 10-\mathrm{H} 10 \mathrm{~A}$ & $111.5(19)$ \\
\hline $\mathrm{C} 6-\mathrm{C} 5-\mathrm{H} 5$ & $121(2)$ & $\mathrm{O} 1-\mathrm{C} 10-\mathrm{H} 10 \mathrm{~B}$ & $108(2)$ \\
\hline $\mathrm{N} 2-\mathrm{C} 6-\mathrm{C} 5$ & $132.6(3)$ & $\mathrm{C} 9-\mathrm{C} 10-\mathrm{H} 10 \mathrm{~B}$ & $112(2)$ \\
\hline $\mathrm{N} 2-\mathrm{C} 6-\mathrm{C} 1$ & $105.5(3)$ & $\mathrm{H} 10 \mathrm{~A}-\mathrm{C} 10-\mathrm{H} 10 \mathrm{~B}$ & $104(3)$ \\
\hline $\mathrm{C} 7-\mathrm{N} 1-\mathrm{C} 1-\mathrm{C} 2$ & $179.9(3)$ & $\mathrm{N} 1-\mathrm{C} 1-\mathrm{C} 6-\mathrm{N} 2$ & $0.5(3)$ \\
\hline
\end{tabular}




\begin{tabular}{llll}
$\mathrm{C} 7-\mathrm{N} 1-\mathrm{C} 1-\mathrm{C} 6$ & $-0.5(3)$ & $\mathrm{C} 2-\mathrm{C} 1-\mathrm{C} 6-\mathrm{C} 5$ & $2.1(4)$ \\
$\mathrm{N} 1-\mathrm{C} 1-\mathrm{C} 2-\mathrm{C} 3$ & $178.5(3)$ & $\mathrm{N} 1-\mathrm{C} 1-\mathrm{C} 6-\mathrm{C} 5$ & $-177.5(3)$ \\
$\mathrm{C} 6-\mathrm{C} 1-\mathrm{C} 2-\mathrm{C} 3$ & $-1.0(4)$ & $\mathrm{C} 1-\mathrm{N} 1-\mathrm{C} 7-\mathrm{N} 2$ & $0.3(3)$ \\
$\mathrm{C} 1-\mathrm{C} 2-\mathrm{C} 3-\mathrm{C} 4$ & $-0.4(5)$ & $\mathrm{C} 1-\mathrm{N} 1-\mathrm{C} 7-\mathrm{C} 8$ & $-177.9(3)$ \\
$\mathrm{C} 2-\mathrm{C} 3-\mathrm{C} 4-\mathrm{C} 5$ & $0.8(5)$ & $\mathrm{C} 6-\mathrm{N} 2-\mathrm{C} 7-\mathrm{N} 1$ & $0.0(3)$ \\
$\mathrm{C} 3-\mathrm{C} 4-\mathrm{C} 5-\mathrm{C} 6$ & $0.2(5)$ & $\mathrm{C} 6-\mathrm{N} 2-\mathrm{C} 7-\mathrm{C} 8$ & $178.2(3)$ \\
$\mathrm{C} 7-\mathrm{N} 2-\mathrm{C} 6-\mathrm{C} 5$ & $177.5(3)$ & $\mathrm{N} 1-\mathrm{C} 7-\mathrm{C} 8-\mathrm{C} 9$ & $-166.7(3)$ \\
$\mathrm{C} 7-\mathrm{N} 2-\mathrm{C} 6-\mathrm{C} 1$ & $-0.3(3)$ & $\mathrm{N} 2-\mathrm{C} 7-\mathrm{C} 8-\mathrm{C} 9$ & $15.3(4)$ \\
$\mathrm{C} 4-\mathrm{C} 5-\mathrm{C} 6-\mathrm{N} 2$ & $-179.1(3)$ & $\mathrm{C} 7-\mathrm{C} 8-\mathrm{C} 9-\mathrm{C} 10$ & $-179.8(3)$ \\
$\mathrm{C} 4-\mathrm{C} 5-\mathrm{C} 6-\mathrm{C} 1$ & $-1.7(5)$ & $\mathrm{C} 8-\mathrm{C} 9-\mathrm{C} 10-\mathrm{O} 1$ & $-178.7(3)$ \\
$\mathrm{C} 2-\mathrm{C} 1-\mathrm{C} 6-\mathrm{N} 2$ & $-179.9(3)$ & & \\
\hline
\end{tabular}

Hydrogen-bond geometry $\left(A,{ }^{\circ}\right)$

\begin{tabular}{lllll}
\hline$D-\mathrm{H} \cdots A$ & $D-\mathrm{H}$ & $\mathrm{H} \cdots A$ & $D \cdots A$ & $D-\mathrm{H}^{\cdots} A$ \\
\hline $\mathrm{N} 2-\mathrm{H} 2 N \cdots \mathrm{O} 1^{\mathrm{i}}$ & $0.84(3)$ & $1.95(3)$ & $2.772(3)$ & $165(3)$ \\
$\mathrm{O} 1-\mathrm{H} 1 \cdots \mathrm{N} 1^{\mathrm{ii}}$ & $0.85(4)$ & $1.90(4)$ & $2.741(3)$ & $169(4)$ \\
$\mathrm{C} 3-\mathrm{H} 3 \cdots \mathrm{C} 3^{\mathrm{iii}}$ & $0.97(4)$ & $2.87(4)$ & $3.770(4)$ & $154(3)$ \\
\hline
\end{tabular}

Symmetry codes: (i) $x+1 / 2,-y+1 / 2,-z+1$; (ii) $-x+1 / 2,-y+1, z+1 / 2$; (iii) $x+1 / 2,-y+1 / 2,-z$.

2-(3-Hydroxypropyl)-1H-benzimidazol-3-ium nitrate (BIZHNO3)

Crystal data

$\mathrm{C}_{10} \mathrm{H}_{13} \mathrm{~N}_{2} \mathrm{O}^{+} \cdot \mathrm{NO}_{3}^{-}$

$M_{r}=239.23$

Monoclinic, $P 2{ }_{1} / n$

$a=8.5100(3) \AA$

$b=8.2525(4) \AA$

$c=16.5130(7) \AA$

$\beta=93.760(4)^{\circ}$

$V=1157.19(9) \AA^{3}$

$Z=4$

\section{Data collection}

Oxford Diffraction Xcalibur, Ruby diffractometer

Radiation source: Enhance $(\mathrm{Cu}) \mathrm{X}$-ray Source

Detector resolution: 10.2576 pixels $\mathrm{mm}^{-1}$

$\omega$ scans

Absorption correction: multi-scan

(CrysAlisPro; Oxford Diffraction, 2009)

$T_{\min }=0.739, T_{\max }=1.000$

\section{Refinement}

Refinement on $F^{2}$

Least-squares matrix: full

$R\left[F^{2}>2 \sigma\left(F^{2}\right)\right]=0.044$

$w R\left(F^{2}\right)=0.128$

$S=1.04$

2345 reflections

167 parameters

0 restraints
$F(000)=504$

$D_{\mathrm{x}}=1.373 \mathrm{Mg} \mathrm{m}^{-3}$

$\mathrm{Cu} K \alpha$ radiation, $\lambda=1.54184 \AA$

Cell parameters from 1670 reflections

$\theta=5.2-75.5^{\circ}$

$\mu=0.91 \mathrm{~mm}^{-1}$

$T=293 \mathrm{~K}$

Block, colorless

$0.12 \times 0.10 \times 0.08 \mathrm{~mm}$

4122 measured reflections

2345 independent reflections

1644 reflections with $I>2 \sigma(I)$

$R_{\text {int }}=0.021$

$\theta_{\max }=75.8^{\circ}, \theta_{\min }=5.4^{\circ}$

$h=-9 \rightarrow 10$

$k=-10 \rightarrow 8$

$l=-20 \rightarrow 18$

Primary atom site location: difference Fourier map

Secondary atom site location: difference Fourier map

Hydrogen site location: mixed

$\mathrm{H}$ atoms treated by a mixture of independent and constrained refinement 
$w=1 /\left[\sigma^{2}\left(F_{\mathrm{o}}^{2}\right)+(0.0715 P)^{2}+0.0026 P\right]$

where $P=\left(F_{\mathrm{o}}^{2}+2 F_{\mathrm{c}}{ }^{2}\right) / 3$

$(\Delta / \sigma)_{\max }<0.001$

$\Delta \rho_{\max }=0.16$ e $\AA^{-3}$

$\Delta \rho_{\min }=-0.15$ e $\AA^{-3}$

Special details

Geometry. All esds (except the esd in the dihedral angle between two 1.s. planes) are estimated using the full covariance matrix. The cell esds are taken into account individually in the estimation of esds in distances, angles and torsion angles; correlations between esds in cell parameters are only used when they are defined by crystal symmetry. An approximate (isotropic) treatment of cell esds is used for estimating esds involving l.s. planes.

Fractional atomic coordinates and isotropic or equivalent isotropic displacement parameters $\left(\AA^{2}\right)$

\begin{tabular}{|c|c|c|c|c|}
\hline & $x$ & $y$ & $z$ & $U_{\text {iso }} * / U_{\text {eq }}$ \\
\hline O1 & $1.12121(17)$ & $0.57042(19)$ & $0.61912(9)$ & $0.0691(5)$ \\
\hline $\mathrm{H} 1 \mathrm{O}$ & $1.102(4)$ & $0.662(3)$ & $0.6461(18)$ & $0.112(10)^{*}$ \\
\hline $\mathrm{O} 2$ & $0.4689(2)$ & $0.27088(18)$ & $0.74998(8)$ & $0.0732(5)$ \\
\hline $\mathrm{O} 3$ & $0.4922(3)$ & $0.5257(2)$ & $0.74234(10)$ & $0.1045(7)$ \\
\hline $\mathrm{O} 4$ & $0.3822(2)$ & $0.4195(2)$ & $0.84232(10)$ & $0.1011(6)$ \\
\hline N1 & $0.65129(19)$ & $0.34198(19)$ & $0.47832(10)$ & $0.0519(4)$ \\
\hline $\mathrm{H} 1 \mathrm{~N}$ & $0.726(3)$ & $0.374(3)$ & $0.4442(14)$ & $0.075(7)^{*}$ \\
\hline N2 & $0.54243(17)$ & $0.29223(18)$ & $0.59032(9)$ & $0.0477(4)$ \\
\hline $\mathrm{H} 2 \mathrm{~N}$ & $0.527(3)$ & $0.290(3)$ & $0.6412(13)$ & $0.065(6)^{*}$ \\
\hline $\mathrm{C} 1$ & $0.5164(2)$ & $0.2529(2)$ & $0.45731(11)$ & 0.0464 (4) \\
\hline $\mathrm{C} 2$ & $0.4513(3)$ & $0.1965(2)$ & $0.38345(12)$ & $0.0591(5)$ \\
\hline $\mathrm{H} 2$ & 0.498209 & 0.216950 & 0.335175 & $0.071^{*}$ \\
\hline N3 & $0.4465(2)$ & $0.4091(2)$ & $0.77860(10)$ & $0.0616(4)$ \\
\hline $\mathrm{C} 3$ & $0.3151(3)$ & $0.1095(2)$ & $0.38500(13)$ & $0.0663(6)$ \\
\hline $\mathrm{H} 3$ & 0.268665 & 0.069383 & 0.336521 & $0.080^{*}$ \\
\hline $\mathrm{C} 4$ & $0.2433(2)$ & $0.0789(2)$ & 0.45704 (14) & $0.0641(6)$ \\
\hline $\mathrm{H} 4$ & 0.149566 & 0.020639 & 0.455243 & $0.077^{*}$ \\
\hline $\mathrm{C} 5$ & $0.3081(2)$ & $0.1330(2)$ & $0.53068(12)$ & $0.0541(5)$ \\
\hline H5 & 0.261444 & 0.111399 & 0.578915 & $0.065^{*}$ \\
\hline C6 & 0.44674 (19) & $0.22143(19)$ & $0.52923(10)$ & 0.0444 (4) \\
\hline $\mathrm{C} 7$ & $0.6645(2)$ & $0.3629(2)$ & $0.55826(11)$ & $0.0486(4)$ \\
\hline $\mathrm{C} 8$ & $0.7967(2)$ & $0.4460(2)$ & $0.60431(12)$ & $0.0576(5)$ \\
\hline $\mathrm{H} 8 \mathrm{~A}$ & 0.765574 & 0.471662 & 0.658317 & $0.069^{*}$ \\
\hline H8B & 0.819160 & 0.547104 & 0.577420 & $0.069^{*}$ \\
\hline C9 & 0.9449 (2) & $0.3426(2)$ & $0.61118(13)$ & $0.0567(5)$ \\
\hline H9A & 0.921976 & 0.240986 & 0.637422 & $0.068^{*}$ \\
\hline H9B & 0.976665 & 0.318146 & 0.557167 & $0.068^{*}$ \\
\hline $\mathrm{C} 10$ & $1.0784(2)$ & $0.4255(3)$ & $0.65864(12)$ & $0.0618(5)$ \\
\hline H10A & 1.047114 & 0.450854 & 0.712636 & $0.074 *$ \\
\hline H10B & 1.168414 & 0.353286 & 0.664051 & $0.074 *$ \\
\hline
\end{tabular}

Extinction correction: SHELXL2016/6

(Sheldrick 2015),

$\mathrm{Fc}^{*}=\mathrm{kFc}\left[1+0.001 \times \mathrm{Fc}^{2} \lambda^{3} / \sin (2 \theta)\right]^{-1 / 4}$

Extinction coefficient: 0.0075 (11) 
Atomic displacement parameters $\left(\AA^{2}\right)$

\begin{tabular}{lllllll}
\hline & $U^{11}$ & $U^{22}$ & $U^{33}$ & $U^{12}$ & $U^{13}$ & $U^{23}$ \\
\hline O1 & $0.0677(10)$ & $0.0726(10)$ & $0.0693(9)$ & $-0.0224(8)$ & $0.0217(7)$ & $-0.0162(8)$ \\
O2 & $0.1068(13)$ & $0.0561(8)$ & $0.0580(8)$ & $0.0013(8)$ & $0.0154(8)$ & $-0.0021(7)$ \\
O3 & $0.1610(19)$ & $0.0591(10)$ & $0.0956(13)$ & $-0.0156(11)$ & $0.0260(13)$ & $0.0054(9)$ \\
O4 & $0.1175(15)$ & $0.1153(15)$ & $0.0749(11)$ & $0.0069(12)$ & $0.0408(10)$ & $-0.0192(10)$ \\
N1 & $0.0507(9)$ & $0.0494(8)$ & $0.0569(9)$ & $-0.0031(7)$ & $0.0136(7)$ & $0.0017(7)$ \\
N2 & $0.0479(8)$ & $0.0489(8)$ & $0.0468(8)$ & $-0.0003(6)$ & $0.0071(7)$ & $-0.0006(7)$ \\
C1 & $0.0469(9)$ & $0.0403(8)$ & $0.0521(9)$ & $0.0048(7)$ & $0.0040(7)$ & $0.0032(7)$ \\
C2 & $0.0714(13)$ & $0.0556(11)$ & $0.0498(10)$ & $0.0078(10)$ & $-0.0003(9)$ & $0.0008(9)$ \\
N3 & $0.0658(11)$ & $0.0633(10)$ & $0.0556(10)$ & $0.0006(8)$ & $0.0026(8)$ & $-0.0041(8)$ \\
C3 & $0.0695(14)$ & $0.0583(12)$ & $0.0681(13)$ & $0.0051(10)$ & $-0.0170(11)$ & $-0.0093(10)$ \\
C4 & $0.0478(11)$ & $0.0521(11)$ & $0.0911(16)$ & $-0.0028(9)$ & $-0.0055(10)$ & $-0.0061(10)$ \\
C5 & $0.0473(10)$ & $0.0466(10)$ & $0.0691(12)$ & $-0.0012(8)$ & $0.0096(9)$ & $0.0020(9)$ \\
C6 & $0.0433(9)$ & $0.0393(8)$ & $0.0509(9)$ & $0.0033(7)$ & $0.0041(7)$ & $0.0011(7)$ \\
C7 & $0.0453(9)$ & $0.0427(9)$ & $0.0582(10)$ & $0.0022(7)$ & $0.0057(8)$ & $-0.0029(8)$ \\
C8 & $0.0489(11)$ & $0.0524(10)$ & $0.0715(13)$ & $-0.0021(8)$ & $0.0041(9)$ & $-0.0132(9)$ \\
C9 & $0.0548(11)$ & $0.0457(9)$ & $0.0685(11)$ & $0.0003(8)$ & $-0.0027(9)$ & $-0.0031(9)$ \\
C10 & $0.0558(11)$ & $0.0662(12)$ & $0.0622(12)$ & $0.0003(10)$ & $-0.0053(9)$ & $-0.0056(10)$ \\
& & & & & &
\end{tabular}

Geometric parameters $\left(\AA,{ }^{\circ}\right)$

\begin{tabular}{llll}
\hline $\mathrm{O} 1-\mathrm{C} 10$ & $1.421(2)$ & $\mathrm{C} 3-\mathrm{C} 4$ & $1.396(3)$ \\
$\mathrm{O} 1-\mathrm{H} 1 \mathrm{C}$ & $0.90(3)$ & $\mathrm{C}-\mathrm{H} 3$ & 0.9300 \\
$\mathrm{O} 2-\mathrm{N} 3$ & $1.254(2)$ & $\mathrm{C} 4-\mathrm{C} 5$ & $1.376(3)$ \\
$\mathrm{O} 3-\mathrm{N} 3$ & $1.211(2)$ & $\mathrm{C} 4-\mathrm{H} 4$ & 0.9300 \\
$\mathrm{O} 4-\mathrm{N} 3$ & $1.220(2)$ & $\mathrm{C} 5-\mathrm{C} 6$ & $1.389(2)$ \\
$\mathrm{N} 1-\mathrm{C} 7$ & $1.329(2)$ & $\mathrm{C} 5-\mathrm{H} 5$ & 0.9300 \\
$\mathrm{~N} 1-\mathrm{C} 1$ & $1.388(2)$ & $\mathrm{C} 7-\mathrm{C} 8$ & $1.484(2)$ \\
$\mathrm{N} 1-\mathrm{H} 1 \mathrm{~N}$ & $0.91(2)$ & $\mathrm{C} 8-\mathrm{C} 9$ & $1.521(3)$ \\
$\mathrm{N} 2-\mathrm{C} 7$ & $1.331(2)$ & $\mathrm{C} 8-\mathrm{H} 8 \mathrm{~A}$ & 0.9700 \\
$\mathrm{~N} 2-\mathrm{C} 6$ & $1.384(2)$ & $\mathrm{C} 8-\mathrm{H} 8 \mathrm{~B}$ & 0.9700 \\
$\mathrm{~N} 2-\mathrm{H} 2 \mathrm{~N}$ & $0.86(2)$ & $\mathrm{C} 9-\mathrm{C} 10$ & $1.502(3)$ \\
$\mathrm{C} 1-\mathrm{C} 6$ & $1.386(2)$ & $\mathrm{C} 9-\mathrm{H} 9 \mathrm{~A}$ & 0.9700 \\
$\mathrm{C} 1-\mathrm{C} 2$ & $1.387(3)$ & $\mathrm{C} 9-\mathrm{H} 9 \mathrm{~B}$ & 0.9700 \\
$\mathrm{C} 2-\mathrm{C} 3$ & $1.365(3)$ & $\mathrm{C} 10-\mathrm{H} 10 \mathrm{~A}$ & 0.9700 \\
$\mathrm{C} 2-\mathrm{H} 2$ & 0.9300 & $\mathrm{C} 10-\mathrm{H} 10 \mathrm{~B}$ & 0.9700 \\
& & & $106.33(15)$ \\
$\mathrm{C} 10-\mathrm{O} 1-\mathrm{H} 1 \mathrm{~N}$ & $114.7(19)$ & $\mathrm{N} 2-\mathrm{C} 6-\mathrm{C} 1$ & $132.01(17)$ \\
$\mathrm{C} 7-\mathrm{N} 1-\mathrm{C} 1$ & $109.37(16)$ & $\mathrm{N} 2-\mathrm{C} 6-\mathrm{C} 5$ & $121.66(17)$ \\
$\mathrm{C} 7-\mathrm{N} 1-\mathrm{H} 1 \mathrm{~N}$ & $124.0(14)$ & $\mathrm{C} 1-\mathrm{C} 6-\mathrm{C} 5$ & $108.73(16)$ \\
$\mathrm{C} 1-\mathrm{N} 1-\mathrm{H} 1 \mathrm{~N}$ & $126.4(14)$ & $\mathrm{N} 1-\mathrm{C} 7-\mathrm{N} 2$ & $125.51(17)$ \\
$\mathrm{C} 7-\mathrm{N} 2-\mathrm{C} 6$ & $109.41(15)$ & $\mathrm{N} 1-\mathrm{C} 7-\mathrm{C} 8$ & $125.72(17)$ \\
$\mathrm{C} 7-\mathrm{N} 2-\mathrm{H} 2 \mathrm{~N}$ & $125.0(15)$ & $\mathrm{N} 2-\mathrm{C} 7-\mathrm{C} 8$ & $112.10(15)$ \\
$\mathrm{C} 6-\mathrm{N} 2-\mathrm{H} 2 \mathrm{~N}$ & $125.6(15)$ & $\mathrm{C} 7-\mathrm{C} 8-\mathrm{C} 9$ & 109.2 \\
$\mathrm{C} 6-\mathrm{C} 1-\mathrm{C} 2$ & $121.46(17)$ & $\mathrm{C} 7-\mathrm{C} 8-\mathrm{H} 8 \mathrm{~A}$ & 109.2 \\
$\mathrm{C} 6-\mathrm{C} 1-\mathrm{N} 1$ & $106.16(15)$ & $\mathrm{C} 9-\mathrm{C} 8-\mathrm{H} 8 \mathrm{~A}$ &
\end{tabular}




\begin{tabular}{|c|c|c|c|}
\hline $\mathrm{C} 2-\mathrm{C} 1-\mathrm{N} 1$ & $132.37(18)$ & $\mathrm{C} 7-\mathrm{C} 8-\mathrm{H} 8 \mathrm{~B}$ & 109.2 \\
\hline $\mathrm{C} 3-\mathrm{C} 2-\mathrm{C} 1$ & $116.80(19)$ & $\mathrm{C} 9-\mathrm{C} 8-\mathrm{H} 8 \mathrm{~B}$ & 109.2 \\
\hline $\mathrm{C} 3-\mathrm{C} 2-\mathrm{H} 2$ & 121.6 & $\mathrm{H} 8 \mathrm{~A}-\mathrm{C} 8-\mathrm{H} 8 \mathrm{~B}$ & 107.9 \\
\hline $\mathrm{C} 1-\mathrm{C} 2-\mathrm{H} 2$ & 121.6 & $\mathrm{C} 10-\mathrm{C} 9-\mathrm{C} 8$ & $112.27(16)$ \\
\hline $\mathrm{O} 3-\mathrm{N} 3-\mathrm{O} 4$ & $123.2(2)$ & $\mathrm{C} 10-\mathrm{C} 9-\mathrm{H} 9 \mathrm{~A}$ & 109.2 \\
\hline $\mathrm{O} 3-\mathrm{N} 3-\mathrm{O} 2$ & $118.34(18)$ & $\mathrm{C} 8-\mathrm{C} 9-\mathrm{H} 9 \mathrm{~A}$ & 109.2 \\
\hline $\mathrm{O} 4-\mathrm{N} 3-\mathrm{O} 2$ & $118.47(18)$ & $\mathrm{C} 10-\mathrm{C} 9-\mathrm{H} 9 \mathrm{~B}$ & 109.2 \\
\hline $\mathrm{C} 2-\mathrm{C} 3-\mathrm{C} 4$ & $122.06(19)$ & С $8-\mathrm{C} 9-\mathrm{H} 9 \mathrm{~B}$ & 109.2 \\
\hline $\mathrm{C} 2-\mathrm{C} 3-\mathrm{H} 3$ & 119.0 & $\mathrm{H} 9 \mathrm{~A}-\mathrm{C} 9-\mathrm{H} 9 \mathrm{~B}$ & 107.9 \\
\hline $\mathrm{C} 4-\mathrm{C} 3-\mathrm{H} 3$ & 119.0 & $\mathrm{O} 1-\mathrm{C} 10-\mathrm{C} 9$ & $110.57(17)$ \\
\hline $\mathrm{C} 5-\mathrm{C} 4-\mathrm{C} 3$ & $121.47(19)$ & $\mathrm{O} 1-\mathrm{C} 10-\mathrm{H} 10 \mathrm{~A}$ & 109.5 \\
\hline $\mathrm{C} 5-\mathrm{C} 4-\mathrm{H} 4$ & 119.3 & $\mathrm{C} 9-\mathrm{C} 10-\mathrm{H} 10 \mathrm{~A}$ & 109.5 \\
\hline $\mathrm{C} 3-\mathrm{C} 4-\mathrm{H} 4$ & 119.3 & $\mathrm{O} 1-\mathrm{C} 10-\mathrm{H} 10 \mathrm{~B}$ & 109.5 \\
\hline $\mathrm{C} 4-\mathrm{C} 5-\mathrm{C} 6$ & $116.53(18)$ & $\mathrm{C} 9-\mathrm{C} 10-\mathrm{H} 10 \mathrm{~B}$ & 109.5 \\
\hline $\mathrm{C} 4-\mathrm{C} 5-\mathrm{H} 5$ & 121.7 & $\mathrm{H} 10 \mathrm{~A}-\mathrm{C} 10-\mathrm{H} 10 \mathrm{~B}$ & 108.1 \\
\hline $\mathrm{C} 6-\mathrm{C} 5-\mathrm{H} 5$ & 121.7 & & \\
\hline $\mathrm{C} 7-\mathrm{N} 1-\mathrm{C} 1-\mathrm{C} 6$ & $0.46(19)$ & $\mathrm{N} 1-\mathrm{C} 1-\mathrm{C} 6-\mathrm{C} 5$ & $-179.68(15)$ \\
\hline $\mathrm{C} 7-\mathrm{N} 1-\mathrm{C} 1-\mathrm{C} 2$ & $-178.67(19)$ & $\mathrm{C} 4-\mathrm{C} 5-\mathrm{C} 6-\mathrm{N} 2$ & $-179.65(17)$ \\
\hline $\mathrm{C} 6-\mathrm{C} 1-\mathrm{C} 2-\mathrm{C} 3$ & $0.4(3)$ & $\mathrm{C} 4-\mathrm{C} 5-\mathrm{C} 6-\mathrm{C} 1$ & $-0.3(3)$ \\
\hline $\mathrm{N} 1-\mathrm{C} 1-\mathrm{C} 2-\mathrm{C} 3$ & $179.40(19)$ & $\mathrm{C} 1-\mathrm{N} 1-\mathrm{C} 7-\mathrm{N} 2$ & $-0.6(2)$ \\
\hline $\mathrm{C} 1-\mathrm{C} 2-\mathrm{C} 3-\mathrm{C} 4$ & $0.4(3)$ & $\mathrm{C} 1-\mathrm{N} 1-\mathrm{C} 7-\mathrm{C} 8$ & $177.29(16)$ \\
\hline $\mathrm{C} 2-\mathrm{C} 3-\mathrm{C} 4-\mathrm{C} 5$ & $-1.1(3)$ & $\mathrm{C} 6-\mathrm{N} 2-\mathrm{C} 7-\mathrm{N} 1$ & $0.50(19)$ \\
\hline $\mathrm{C} 3-\mathrm{C} 4-\mathrm{C} 5-\mathrm{C} 6$ & $1.0(3)$ & $\mathrm{C} 6-\mathrm{N} 2-\mathrm{C} 7-\mathrm{C} 8$ & $-177.38(16)$ \\
\hline $\mathrm{C} 7-\mathrm{N} 2-\mathrm{C} 6-\mathrm{C} 1$ & $-0.21(18)$ & $\mathrm{N} 1-\mathrm{C} 7-\mathrm{C} 8-\mathrm{C} 9$ & $-74.5(2)$ \\
\hline $\mathrm{C} 7-\mathrm{N} 2-\mathrm{C} 6-\mathrm{C} 5$ & $179.26(18)$ & $\mathrm{N} 2-\mathrm{C} 7-\mathrm{C} 8-\mathrm{C} 9$ & $103.1(2)$ \\
\hline $\mathrm{C} 2-\mathrm{C} 1-\mathrm{C} 6-\mathrm{N} 2$ & $179.09(16)$ & $\mathrm{C} 7-\mathrm{C} 8-\mathrm{C} 9-\mathrm{C} 10$ & $-179.28(17)$ \\
\hline $\mathrm{N} 1-\mathrm{C} 1-\mathrm{C} 6-\mathrm{N} 2$ & $-0.15(18)$ & $\mathrm{C} 8-\mathrm{C} 9-\mathrm{C} 10-\mathrm{O} 1$ & $-62.3(2)$ \\
\hline $\mathrm{C} 2-\mathrm{C} 1-\mathrm{C} 6-\mathrm{C} 5$ & $-0.4(3)$ & & \\
\hline
\end{tabular}

Hydrogen-bond geometry $\left(A,{ }^{\circ}\right)$

\begin{tabular}{lllll}
\hline$D-\mathrm{H} \cdots A$ & $D-\mathrm{H}$ & $\mathrm{H} \cdots A$ & $D \cdots A$ & $D-\mathrm{H} \cdots A$ \\
\hline $\mathrm{N} 2-\mathrm{H} 2 N \cdots \mathrm{O} 2$ & $0.86(2)$ & $1.90(2)$ & $2.755(2)$ & $173(2)$ \\
$\mathrm{N} 1-\mathrm{H} 1 N \cdots \mathrm{O} 1^{\mathrm{i}}$ & $0.91(2)$ & $1.78(2)$ & $2.696(2)$ & $177(2)$ \\
$\mathrm{O} 1-\mathrm{H} 1 O \cdots \mathrm{O} 2^{2 i}$ & $0.90(3)$ & $2.06(3)$ & $2.866(2)$ & $149(3)$ \\
$\mathrm{O} 1-\mathrm{H} 1 O \cdots \mathrm{O} 4^{\mathrm{ii}}$ & $0.90(3)$ & $2.14(3)$ & $2.951(3)$ & $150(3)$ \\
$\mathrm{C} 5-\mathrm{H} 5 \cdots \mathrm{O} 4^{\mathrm{iii}}$ & 0.93 & 2.43 & $3.251(3)$ & 147
\end{tabular}

Symmetry codes: (i) $-x+2,-y+1,-z+1$; (ii) $-x+3 / 2, y+1 / 2,-z+3 / 2$; (iii) $-x+1 / 2, y-1 / 2,-z+3 / 2$. 